\title{
The genesis of Typhoon Nuri as observed during the Tropical Cyclone Structure 2008 (TCS08) field experiment - Part 2: Observations of the convective environment
}

\author{
M. T. Montgomery ${ }^{1}$ and R. K. Smith ${ }^{2}$ \\ ${ }^{1}$ Department of Meteorology, Naval Postgraduate School, Monterey, CA \& NOAA’s Hurricane Research Division, Miami, \\ FL, USA \\ ${ }^{2}$ Meteorological Institute, University of Munich, Munich, Germany \\ Correspondence to: M. T. Montgomery (mtmontgo@ nps.edu)
}

Received: 11 August 2011 - Published in Atmos. Chem. Phys. Discuss.: 23 November 2011

Revised: 14 February 2012 - Accepted: 22 March 2012 - Published: 4 May 2012

\begin{abstract}
Analyses of thermodynamic data gathered from airborne dropwindsondes during the Tropical Cyclone Structure (2008) experiment are presented for the disturbance that became Typhoon Nuri. Although previous work has suggested that Nuri formed within the protective recirculating "pouch" region of a westward propagating wave-like disturbance and implicated rotating deep convective clouds in driving the inflow to spin up the tangential circulation of the system-scale flow, the nature of the thermodynamic environment that supported the genesis remains a topic of debate.

During the genesis phase, vertical profiles of virtual potential temperature show little variability between soundings on a particular day and the system-average soundings likewise show a negligible change. There is a tendency also for the lower and middle troposphere to moisten. However, the data show that, on the scale of the recirculating region of the disturbance, there was no noticeable reduction of virtual temperature in the lower troposphere, but a small warming (less than $1 \mathrm{~K}$ ) in the upper troposphere. Vertical profiles of pseudo-equivalent potential temperature, $\theta_{\mathrm{e}}$, during the genesis show a modestly decreasing deficit of $\theta_{\mathrm{e}}$ in the vertical between the surface and the height of minimum $\theta_{\mathrm{e}}$ (between 3 and $4 \mathrm{~km}$ ), from $17.5 \mathrm{~K}$ to $15.2 \mathrm{~K}$.

The findings reported here are consistent with those found for developing disturbances observed in the Pre-Depression Investigation of Cloud Systems in the Tropics (PREDICT) experiment in 2010. Some implications of the findings are discussed.
\end{abstract}

\section{Introduction}

Understanding the dynamics and thermodynamics of tropical cyclogenesis remains one of the great unsolved problems in tropical meteorology. One reason for the lack of understanding is undoubtedly the fact that genesis occurs over the tropical oceans where conventional observational data such as radar data, radiosonde soundings and surface data are relatively sparse. While there have been a few field experiments directed at documenting cyclogenesis (Bister and Emanuel, 1997; Ritchie and Holland, 1999) and a few serendipitous sets of measurements (Reasor et al., 2005; Houze et al., 2009), many questions remain about the processes involved and their relative importance. A recent review of work over the past few years is given by Montgomery and Smith (2010).

One prominent study of tropical cyclogenesis in the eastern Pacific basin was that of Bister and Emanuel (1997; hereafter BE97), which was an outcome of the TEXMEX experiment. This study emphasized the importance of thermodynamical processes within a so-called "mesoscale convective vortex embryo". The study proposed that the development of a cool, moist environment resulting from stratiform rain serves as the incubation region for the formation of a lowlevel, warm-core cyclonic vortex. The study suggested that sustained precipitation in the stratiform cloud deck together with the evaporation of rain drops below would gradually cool and saturate the layer below cloud base while transporting cyclonic vorticity downwards to the surface. The idea is that there will be an accompanying increase in near surface winds that would increase surface moisture fluxes leading 
ultimately to convective destabilization. A subsequent bout of deep convection was hypothesized to induce low-level convergence and vorticity stretching, thereby increasing the low-level tangential winds and "igniting" an amplification process fuelled by the increased surface moisture fluxes.

Some questions about the dynamics of the pre-ignition process have been raised by Tory and Montgomery (2006) who noted, in particular, the inconsistency with vorticity substance impermeability between isobaric surfaces (Haynes and McIntrye, 1987). An equivalent way to understand this inconsistency is through the use of absolute angular momentum. The downward advection of absolute angular momentum by the downdraught is accompanied by horizontal divergence, which moves absolute angular momentum surfaces outwards. The net effect of this process is one in which the absolute angular momentum is materially conserved. Since the absolute circulation is proportional to the absolute angular momentum, the absolute circulation similarly will not change. Thus the hypothesized mechanism cannot increase the absolute circulation of the lower troposphere and cannot lead to a net spin up of the low-level circulation. Recent work has raised concerns also about the assumed air-sea interaction feedback, which are discussed and appraised by Montgomery et al. (2009). Notwithstanding these issues, the thermodynamical aspects of the genesis process are still interesting and worthy of investigation using the data analysed in Sect. 3.

Influenced by the results of the TEXMEX experiment, Raymond and Sessions (2007) proposed an alternative thermodynamic view of tropical cyclogenesis that is linked with the changes of the thermal stability of the tropical atmosphere ("warming at upper levels and cooling at low levels", their page 3 , right column). They simulated a radiativeconvective equilibrium state in the absence of ambient rotation and in a two-dimensional (zonal-height) cloud-resolving model. Relative to this equilibrium state, they found that perturbed environments which were about $1 \mathrm{~K}$ cooler at low levels and about $1 \mathrm{~K}$ warmer at upper levels lowered the level of maximum vertical mass flux from $10 \mathrm{~km}$ to approximately $5 \mathrm{~km}$. They found also that this stabilization effect was more effective than a humidification of the column. Specifically, their numerical results suggested that the stabilization of the column "... results in a concentration of the inflow into a shallower layer, which produces more low-level vorticity convergence per unit rainfall. It is thus even more effective than moisturization in aiding the spinup of a tropical cyclone according to our simulations". The suggestion is that if realistic values of ambient rotation associated with a tropical wave or monsoon trough were included, this inflow would cause a stronger vorticity convergence at lower levels than at mid-levels and thus contribute to the spin up of the system. Raymond and Sessions suggest this mechanism as a possible explanation for why tropical-wave-scale mid-level vortices foster tropical storm formation. This is an idea that has been challenged by Dunkerton et al. (2009), who proposed an alternative view of the problem as discussed below.

In the late summer of 2008, a field experiment, called Tropical Cyclone Structure (TCS08), was conducted by the US Office of Naval Research in the Northwestern Pacific basin. The TCS08 experiment took place from August through September 2008 and its overall objectives were to develop a better understanding of storm-scale processes in the western North Pacific associated with tropical cyclones and to further the understanding of interactions between convective processes and tropical cyclone genesis, structure/evolution, intensity, and predictability (Elsberry and Harr, 2008). For the component of the experiment that focused on the process of tropical-cyclone formation, priority was given to developing storms before their classification as a tropical depression ${ }^{1}$.

To accomplish the objectives of the TCS08 experiment, a multi-platform observing strategy was implemented. Both United States Air Force (USAF) C130 Hurricane Hunter aircraft [providing dropwindsondes, flight-level data, Stepped Frequency Microwave Radiometer (SFMR) data, and the ability to drop both Airborne Expendable Bathometry Thermographs (AXBT) and ocean buoys] and the Naval Research Laboratory (NRL) P3 aircraft [fitted with the Electra Doppler Radar (ELDORA), dropwindsonde capability, a Doppler wind lidar, and the ability to collect flight-level data] flew aircraft research missions originating from Guam, a USA territory. In the experiment, the USAF C130 was flown at a relatively high altitude of approximately $10 \mathrm{~km}$ in conjunction with the NRL P3 flying at lower altitudes between 3 and $5 \mathrm{~km}$. For further information on the observing strategies and scientific objectives of TCS08, see the experimental overview presented by Elsberry and Harr (2008).

The formation of typhoon Nuri was arguably the best observed genesis event during the TCS08 experiment. A summary of the large-scale and mesoscale aspects leading to Nuri's genesis is presented in Montgomery et al. (2010) and Raymond and Lopez (2011). In brief, Montgomery et al. (2010) used global model analyses, global model forecast data, satellite and dropsonde data to suggest that Nuri formed inside the recirculating "cat's eye" region within the critical layer of a westward propagating wave-like disturbance á la Dunkerton et al. (2009). Raymond and Lopez (2011)

\footnotetext{
${ }^{1}$ The glossary on NOAA's Hurricane Research Division website uses "tropical cyclone" as the generic term for a nonfrontal synoptic-scale low-pressure system over tropical or sub-tropical waters with organized convection (i.e. thunderstorm activity) and a definite cyclonic surface wind circulation. Notably, this definition does not invoke any wind threshold. The same glossary defines a "tropical depression" as a tropical cyclone with maximum sustained surface winds of less than $17 \mathrm{~m} \mathrm{~s}^{-1}$ (34 kt, $39 \mathrm{mph}$ ) and, in the Atlantic and Eastern Pacific Basins, a tropical storm as a tropical cyclone with surface winds between $17 \mathrm{~m} \mathrm{~s}^{-1}$ and $33 \mathrm{~m} \mathrm{~s}^{-1}$. In this study we will define genesis as the formation of a tropical depression and we impose no formal threshold on wind speed.
} 
presented a complementary study of the genesis using the ELDORA radar and dropsonde data and they implicated the inflow induced by rotating deep convective clouds in spinning up the tangential circulation of the system-scale flow.

The most recent experiment focusing on tropical cyclogenesis was the Pre-Depression Investigation of Cloud Systems in the Tropics (PREDICT) experiment. The PREDICT experiment was carried out in the Atlantic and Caribbean region in the summer of 2010 (Montgomery et al., 2012) to test, inter alia, the marsupial paradigm of Dunkerton et al. (2009). It was conducted in collaboration with the National Aeronautics and Space Administration's (NASA) Genesis and Rapid Intensification (GRIP) experiment and the National Oceanographic and Atmospheric Administration's (NOAA) Intensity Forecasting (IFEX) experiment. In a recent paper we have examined the thermodynamic characteristics of developing and non-developing disturbances during the PREDICT experiment (Smith and Montgomery, 2012a). A finding germane to the discussion above was that the systemaverage virtual potential temperature $\theta_{\mathrm{v}}$, for both developing and non-developing disturbances showed a slight warming over the observational period. The most prominent difference between the non-developing system studied and the two systems that developed was the much larger reduction of pseudo-equivalent potential temperature, $\theta_{\mathrm{e}}^{2}$, between the surface and a height of $3 \mathrm{~km}$, typically $25 \mathrm{~K}$ in the nondeveloping system, compared with only $17 \mathrm{~K}$ in the systems that developed.

In the light of results from the PREDICT data, it is pertinent to carry out a similar analysis of the sounding data acquired during the development of the disturbance identified as TCS015, which eventually became typhoon Nuri on 18 August 2008. We focus here specifically on the day-to-day changes in the mean profiles of $\theta_{\mathrm{v}}$ and $\theta_{\mathrm{e}}$ near and within the "pouch" region of the developing disturbance. In this work the pouch region is defined as the recirculating flow in the parent wave's critical layer (see Dunkerton et al. (2009) for definition of critical layer and related terms). So as to clarify the meaning of the term pouch herein, the recirculating flow need not be strictly closed. For one thing, because of the small, but not necessarily negligible time dependence of the flow field in the neighbourhood of the pouch defined as above, the transient component of the flow can contribute to an opening of the streamlines near the dividing streamline that passes through the nearest stagnation point in the co-moving frame. For another, the low-level flow near $925 \mathrm{hPa}$ may be convergent, requiring a slightly open pouch even if the flow were steady. Nevertheless, a key ingredient hypothesized for genesis is the recirculating flow as it will tend to provide a favourable environment for moisturization by deep cumulus convection and the aggregation of convectively-generated vorticity seedlings. In view of these

\footnotetext{
${ }^{2}$ The pseudo-equivalent potential temperature was calculated using Bolton's formula (Bolton, 1980).
}

considerations, we will include the nearby recirculating flow (i.e. streamlines that otherwise appear not to enter the pouch) in our targeted region of interest for all quantitative analyses. The analyses presented will thus provide quantitative information on the changes of stability and moisture in the thermodynamic mean state of the critical layer region of the disturbance that became Nuri.

An outline of the remaining parts of this paper is as follows. Section 2 reviews the sequence of events during the formation and subsequent intensification of typhoon Nuri and describes the dropwindsonde data and methods of analysis. Section 3 presents the results of the analyses for the Nuri disturbance. Section 4 provides a discussion of the results. The conclusions are given in Sect. 5 .

\section{Dropsonde observations of the formation of typhoon Nuri}

Typhoon Nuri was the first tropical cyclogenesis event occurring within aircraft observational range $(3000 \mathrm{~km})$ during TCS08. The storm was flown on four consecutive days (16-19 August) by the USAF C130, enabling the documentation of the evolution from an easterly wave to a Category 3 typhoon. The NRL P3 flew concurrently with the C130 on three days (16-18 August), enabling a documentation of the mesoscale and convective structure during the transition from easterly wave to tropical storm (TS) intensity. The best track data for the period of interest is shown in Fig. 5 of Montgomery et al. (2010) and Fig. 2 of Raymond and LopezCarillo (2011).

The four sets of flights into Nuri took place between 1516 August, on 16 August, between 17-18 August and on 19 August (see Fig. 1 of Montgomery et al., 2010). Following Raymond and Lopez (2010), we refer to the state of Nuri observed during these times as Nuri1, Nuri2, Nuri3 and Nuri4, respectively. As described therein and also in Montgomery et al. (2010), the genesis occurred between Nuri1 and Nuri2 when the disturbance was declared a tropical depression by Joint Typhoon Warning Center forecasters. After quality control using the NCAR Aspen software and further manual inspection to eliminate sondes that did not record all the way to the surface, the precise number of usable sondes from the two aircraft is summarized here. Altogether, there were 37 usable sondes dropped in Nuri1 (22 from the C130, 15 from the P3); 44 in Nuri2 (30 from the C130, 14 from the P3); 29 in Nuri3 (9 from the C130, 20 from the P3); and 24 in Nuri4, all from the $\mathrm{C} 130$. The drop locations, flight tracks and surface pressures measured by the sondes are shown in Fig. $1^{3}$. Many of the sondes dropped from the C130 during Nuri1

\footnotetext{
${ }^{3}$ During Nuri1, the time gap in the C130 track (between 17:54 UTC and 22:21 UTC) is explained by the fact that the first C130 had mechanical problems after releasing the first three dropsondes and had to return to base on Guam. The mission was continued approximately two hours later by a second C130 aircraft, which was tasked
} 

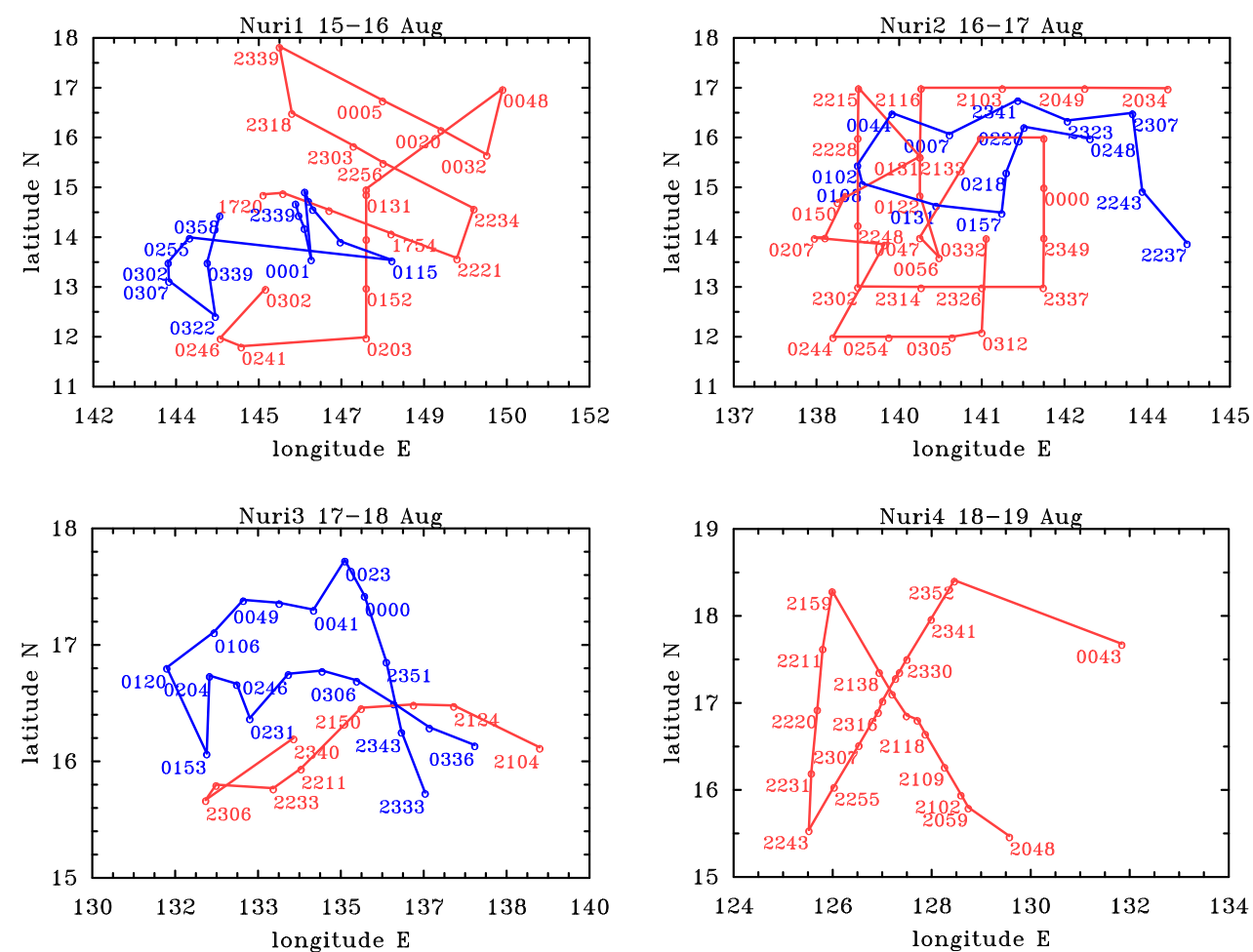

Fig. 1. Drop points and times of drops for (a) Nuri1, (b) Nuri2, (c) Nuri3, and (d) Nuri4. The aircraft track is marked with the location of soundings along the track (small circles). Red denotes the C130 aircraft while blue denotes the NRL P3 aircraft. See text for further details.

and Nuri2 were from about $10 \mathrm{~km}$. The sondes released from the $\mathrm{C} 130$ for Nuri3 were from an altitude of about $7.5 \mathrm{~km}$. During Nuri4, all but two of the sondes were released from an altitude of about $3 \mathrm{~km}$.

In this study we are adopting the perspective of the marsupial paradigm and, unlike Raymond and Lopez (2010) and Raymond et al. (2011), we do not limit our analysis around a particular middle-level circulation centre within the disturbance and its partial overlap with a surface circulation. While we agree with Raymond and Lopez op. cit. that the vertical alignment of the pouch was an important element in Nuri's genesis (see also Montgomery et al., 2010), our perspective here is a broader one that is focused on the entire pouch region (that includes not only the region bounded by the closed streamlines passing through the nearest stagnation point in the co-moving frame, but also the nearby recirculating flow as defined above). With this perspective in mind, the distribution of dropsondes during Nuri1 and Nuri2 is shown in Fig. 2a and b, respectively. The dropsonde locations are superimposed on the $925 \mathrm{hPa}$ streamlines as viewed in the frame of reference moving with the parent wave-like disturbance ( $\approx 7 \mathrm{~m} \mathrm{~s}^{-1}$ westward). The $925 \mathrm{hPa}$ level is chosen here because the parent wave disturbance had its maximum amplitude at or near this level on the first day of observa-

to complete the remaining flight pattern (J. Hawkins and L. Lussier, personal communication, 2011). tion (Montgomery et al., 2010), consistent with prior observations of westward-propagating disturbances in this region (e.g. Reed and Recker, 1971; Chang et al., 1970). The dropsondes and co-moving streamlines are superimposed also on the infrared brightness temperature; the orange and red colors indicate the approximate cloud-top temperatures of areas of active deep convection and their associated anvil clouds.

In order to provide an approximate synthesis of the drop locations relative to the moving pouch region near the genesis time of the disturbance, Fig. 2c shows the distribution of the Nuri1 and Nuri2 dropsondes relative to the co-moving streamlines on the $925 \mathrm{hPa}$ level at 12:00 UTC 16 August $^{4}$. To be synchronous with the $12 \mathrm{Z}$ co-moving streamlines, the zonal phase speed of the parent wave disturbance has been used to translate the drop points for Nuri1 westwards by 3.5 degrees longitude and to translate the drop points for Nuri2 eastwards by 3.5 degrees. The figure indicates clearly that almost all of the drop points are near or within the recirculating region of the disturbance during this time interval and helps substantiate the approach adopted below of taking horizontal averages of all of the usable dropsonde data to typify the thermodynamic environment of the recirculating (pouch) region of the disturbance. It is noteworthy that the fractional

\footnotetext{
${ }^{4}$ The corresponding wind speed distribution at these times in the co-moving frame of reference is shown in Figs. 12 and 14 of Montgomery et al. (2010).
} 

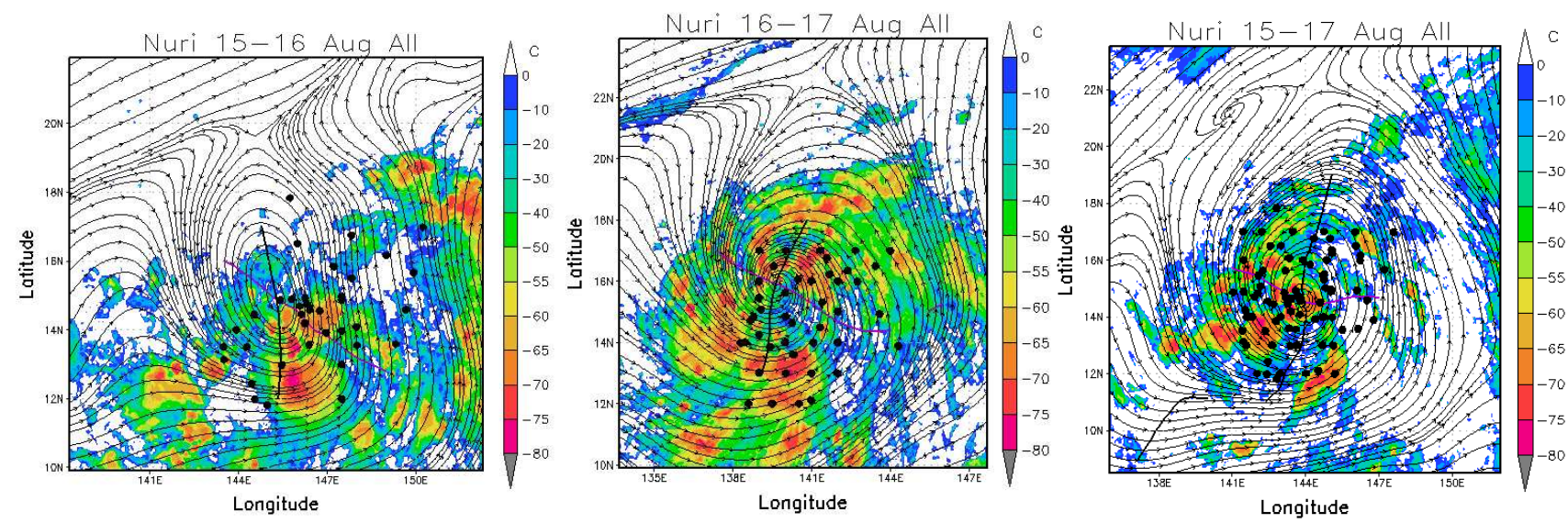

Fig. 2. Dropsonde locations for Nuri1 and Nuri2 as viewed in the co-moving frame of reference with the parent wave-like disturbance (moving approximately $7 \mathrm{~m} \mathrm{~s}^{-1}$ westward). The usable drop points and co-moving streamlines at the $925 \mathrm{hPa}$ level from the GFS FNL analysis are superimposed on the satellite brightness temperature. The color bar for the brightness temperature is indicated. The orange and red colors indicate the approximate cloud-top temperatures of areas of active deep convection and their associated anvil clouds. Shown are (a) dropsonde locations for Nuri1 together with $925 \mathrm{hPa}$ co-moving streamlines and brightness temperature at 00:00 Z 16 August; (b) dropsonde locations for Nuri2 together with $925 \mathrm{hPa}$ co-moving streamlines and brightness temperature at 00:00 Z 17 August; (c) drop locations for both Nuri1 and Nuri2 translated to the 12:00 Z 16 August brightness temperature image and co-moving streamline field, the time nearest the genesis time as declared by Joint Typhoon Warning Center forecasters. The purple curve denotes the local critical latitude where the parent wave's zonal phase speed coincides with the mean zonal flow at the $925 \mathrm{hPa}$ level. The dark black curve denotes the trough axis of the wave disturbance at this same level. The intersection between these two curves denotes the sweet spot of the pouch region (see Montgomery et al. (2010) for further details).

coverage of deep convective activity, as inferred from the fractional area of low cloud-top temperatures, was greater in all quadrants in Nuri2 than in Nuri1.

\section{Results}

Figure 3 shows the vertical structure of $\theta_{\mathrm{v}}$ and $\theta_{\mathrm{e}}$ for all usable soundings on Nuri1, Nuri2, Nuri3 and Nuri4. It shows also the mean profile on each day of measurement and the saturation pseudo-equivalent potential temperature $\left(\theta_{\mathrm{es}}\right)$ based on this mean sounding.

In the genesis phase (between Nuri1 and Nuri2) the vertical structure of $\theta_{\mathrm{v}}$ shows little variability between soundings. This variability increases noticeably between Nuri3 and Nuri4. As a reminder, there were only nine soundings above $3 \mathrm{~km}$ in Nuri3 and only two soundings above $3 \mathrm{~km}$ in Nuri4. The former were dropped en route to the tropical storm and the latter were dropped en route to and from the typhoon and may not be representative of the core region of the system. On all days, the vast majority of the $\theta_{\mathrm{e}}$ curves lie to the left of the $\theta_{\mathrm{es}}$-curve for the mean sounding. The variability in $\theta_{\mathrm{e}}$ between soundings increases significantly between Nuri3 and Nuri4. Part of this variability may be associated with the increasing range of near-surface wind speeds on days 3 and 4 . These characteristics are very similar to those found for developing disturbances during the PREDICT experiment (Smith and Montgomery, 2012a, b).
Figure 4 shows the vertical profiles of relative humidity of all soundings during Nuri1 through Nuri4. These profiles show that the variability found in $\theta_{\mathrm{e}}$ is associated with variations in moisture.

Figure 5 shows vertical profiles of system-averaged $\theta_{\mathrm{v}}, \theta_{\mathrm{e}}$ and relative humidity for all days of Nuri. There is little discernible change in $\theta_{\mathrm{v}}$ below $5 \mathrm{~km}$ during the genesis phase between Nuri1 and Nuri2, but there is a slight warming (not more than $1 \mathrm{~K}$ ) through much of the column between Nuri2 and Nuri3.

As far as $\theta_{\mathrm{e}}$ is concerned, between Nuri1 and Nuri2 there is no significant change below $2 \mathrm{~km}$, but there is a modest increase in $\theta_{\mathrm{e}}$ of about $4 \mathrm{~K}$ that extends to $9 \mathrm{~km}$ altitude. On subsequent days, there is a prominent increase of $\theta_{\mathrm{e}}$ at most levels below $3 \mathrm{~km}$. Above $4 \mathrm{~km}$, however, there is a reduction in $\theta_{\mathrm{e}}$, though this may be an artifact of the small number of sondes at these heights ( 9 for Nuri3 and only 2 for Nuri4). Finally, vertical profiles of $\theta_{\mathrm{e}}$ during the genesis show a modestly decreasing deficit of $\theta_{\mathrm{e}}$ between the surface and the height of minimum $\theta_{\mathrm{e}}$ (between 3 and $4 \mathrm{~km}$ ), from $17.5 \mathrm{~K}$ to $15.2 \mathrm{~K}$.

The changes in relative humidity follow broadly the changes in $\theta_{\mathrm{e}}$. Between Nuri1 and Nuri2, there is no significant change in the relative humidity below $2 \mathrm{~km}$, but there is a noticeable increase above $3 \mathrm{~km}$. Between Nuri2 and Nuri3, the relative humidity increases below $2 \mathrm{~km}$ and decreases above $3 \mathrm{~km}$. Between Nuri3 and Nuri4, the mean relative humidity at low levels does not change appreciably. Above 

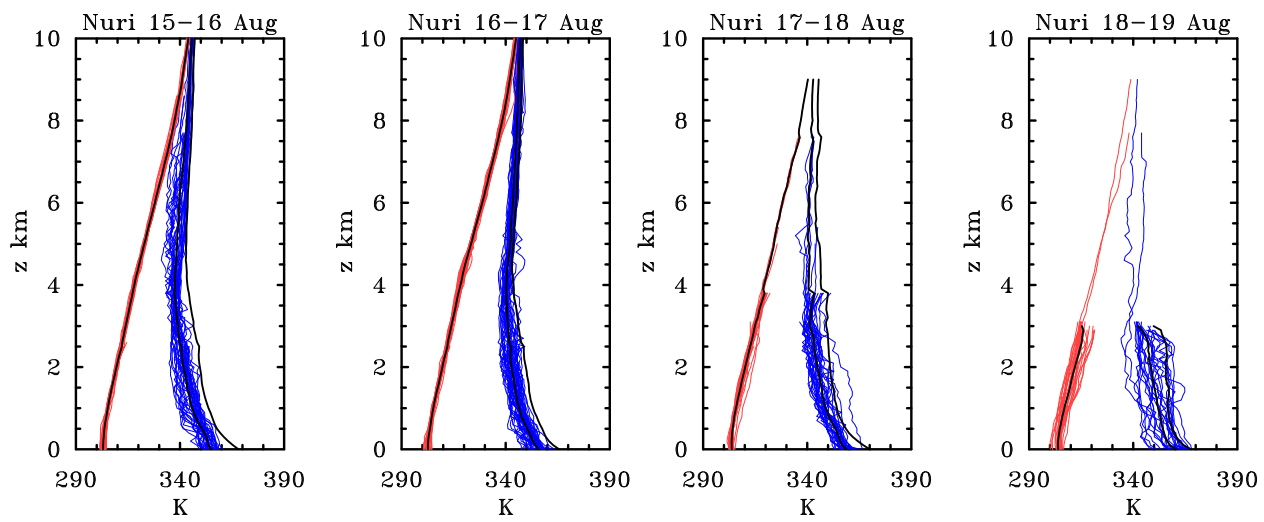

Fig. 3. Vertical profiles of the virtual potential temperature $\left(\theta_{\mathrm{V}}\right)$ and pseudo-equivalent potential temperature $\left(\theta_{\mathrm{e}}\right)$ from each usable sounding on all days of Nuri. Plotted also is the mean profile on each day of measurement (solid black curves) and the saturation pseudo-equivalent potential temperature $\left(\theta_{\mathrm{es}}\right)$ based on this mean sounding (far right solid black curve). The mean sounding for the last day of observations is plotted only up to $3 \mathrm{~km}$ height since the two soundings that extend to higher levels are located outside of the storm. See text for further details.
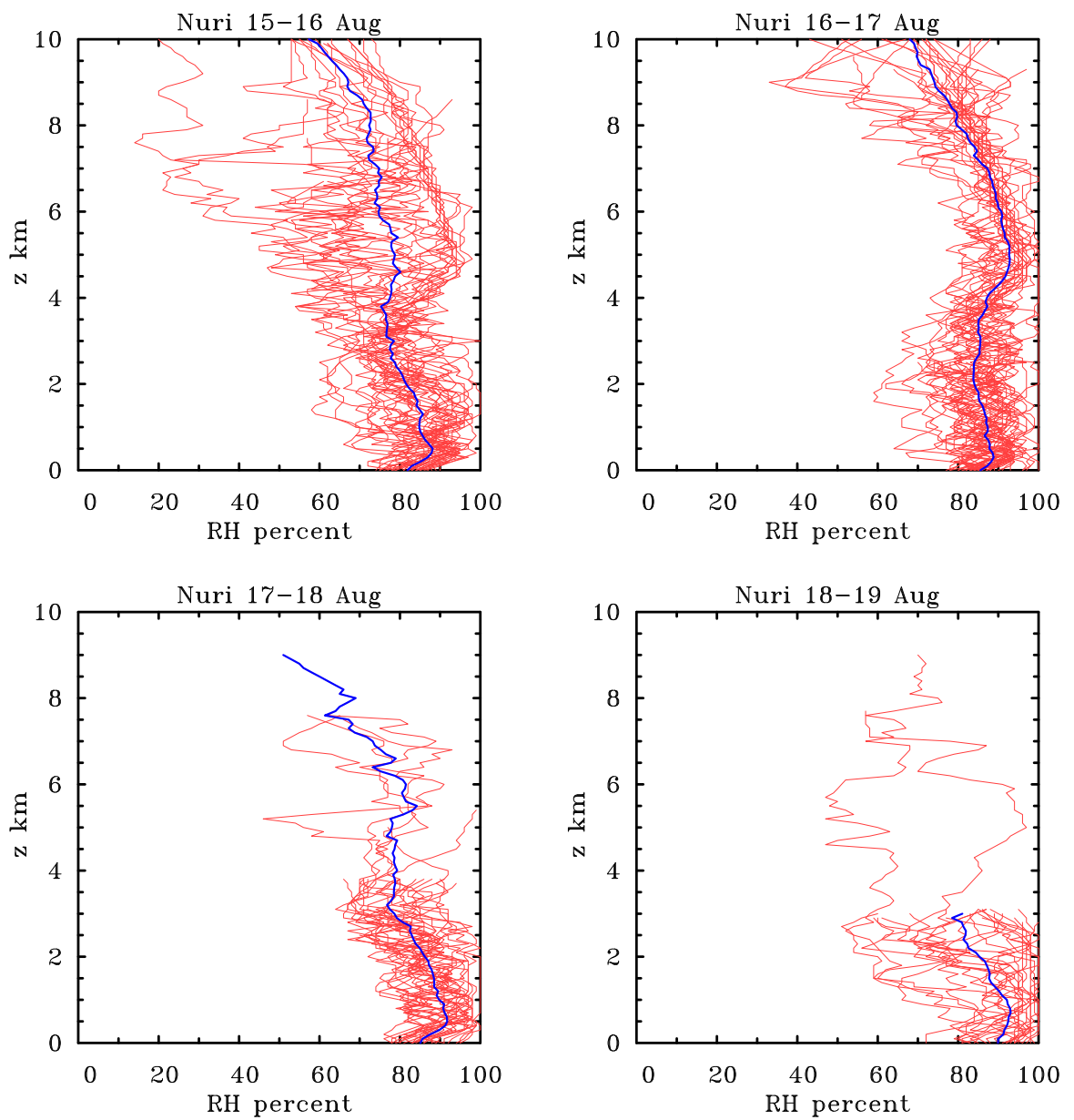

Fig. 4. Vertical profiles of relative humidity $(\mathrm{RH})$ for each usable sounding on all days of Nuri. The system-average RH is depicted by the solid blue curve. The mean sounding for the last day of observations is plotted only up to $3 \mathrm{~km}$ height since the two soundings that extend to higher levels are located outside of the storm. 

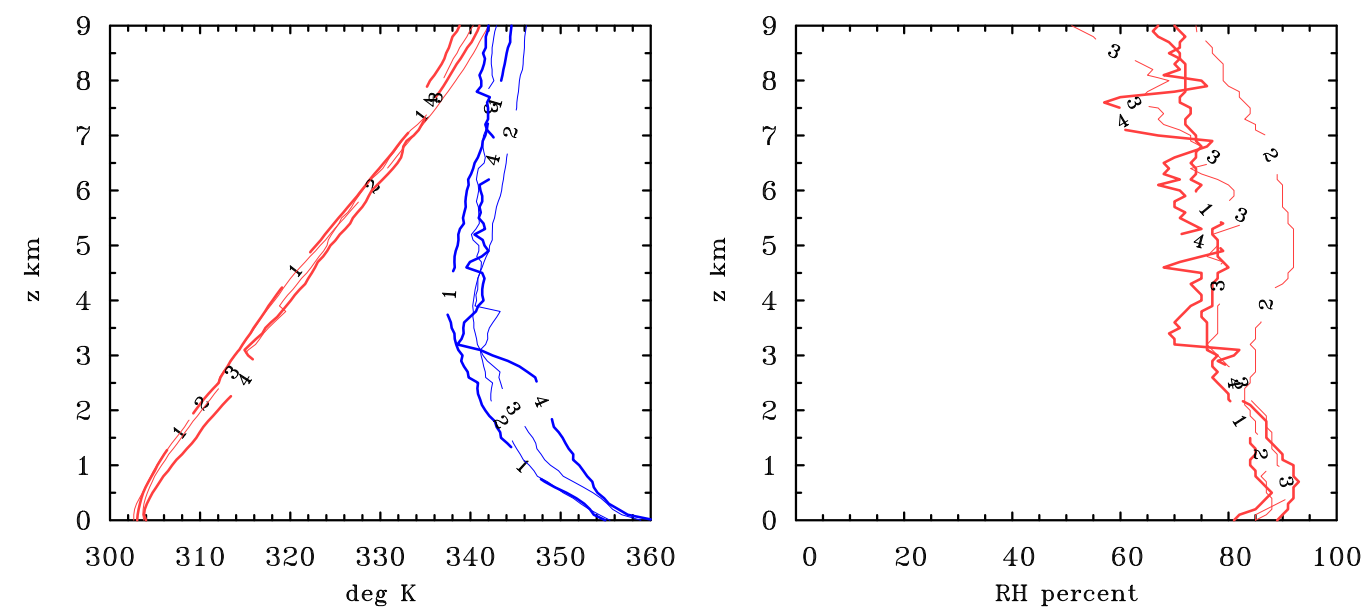

Fig. 5. Vertical profiles of the system-averaged $\theta_{\mathrm{v}}$ and $\theta_{\mathrm{e}}$ (left panel) and RH (right panel) for all four days of Nuri. The thick curves mark the first and last days of the sequence. Numbers on curves refer to the particular day of Nuri: Nuri1, Nuri2, etc. See text for further details.

$3 \mathrm{~km}$ the variability seen may be because of the relatively few soundings noted above.

Figure 6 shows the vertical profile of the difference in the system-averaged virtual temperature between Nuri2 and Nuri1: $\delta \bar{T}_{\mathrm{v}}=\bar{T}_{\mathrm{v}}\left(\right.$ Nuri2) $-\bar{T}_{\mathrm{v}}$ (Nuri1), where the overbar denotes the system average. This quantity is shown in light of the hypothesized thermodynamic control on the cumulus convection and the corresponding convective mass flux discussed by Raymond and colleagues in the Introduction. Note that we are using virtual temperature here as it is the pertinent measure of density for a moist unsaturated air parcel. Apart from a cooling of $0.4 \mathrm{~K}$ between approximately $500 \mathrm{~m}$ and the sea surface, the figure shows no systematic cooling of the lower troposphere (below $4 \mathrm{~km}$ ) during the genesis. The figure shows also that there is a warming of the upper troposphere that increases approximately linearly with height above $4 \mathrm{~km}$. However, the increase in virtual temperature is less than $1 \mathrm{~K}$.

All of the above findings are broadly consistent with those found for developing disturbances observed in the recent PREDICT experiment summarized in the Introduction.

\section{Discussion}

Two notable thermodynamic models have been offered previously to explain tropical cyclogenesis (see Smith and Montgomery, 2012a, Sect. 5). One of these models, that of Raymond and Sessions (2007), has been invoked to help explain the formation of Typhoon Nuri. This model assumes a modest cooling of the lower troposphere in the disturbance region in association with a cyclonic circulation that has a maximum in the middle troposphere. To examine the credibility of this assumption, we calculated the change in the system-mean virtual temperature near the pouch region of Nuri during the genesis phase. In terms of virtual temperature, these calcu-

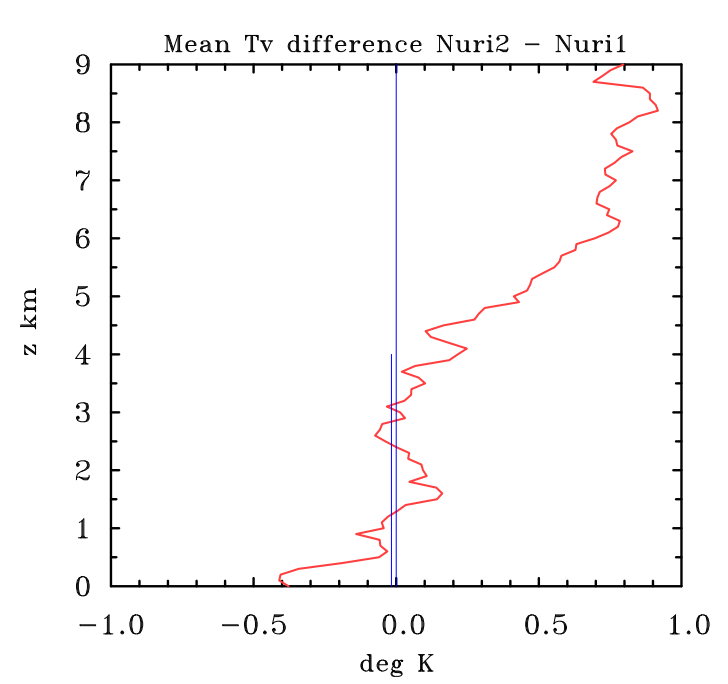

Fig. 6. Vertical profile of the difference in the system-average virtual temperature between Nuri2 and Nuri1: $\delta \bar{T}_{\mathrm{v}}=\bar{T}_{\mathrm{v} 2}-\bar{T}_{\mathrm{v} 1}$, where the overbar denotes the system average. The chosen time interval spans the observed transformation of the pre-Nuri wave-like disturbance into a tropical depression as declared by Joint Typhoon Warning Center forecasters. The thin blue vertical line to the left of the ordinate indicates the related system-averaged temperature difference below $4 \mathrm{~km}$. See text for details.

lations show no systematic cooling of the lower troposphere (below $4 \mathrm{~km}$ ) during the genesis and, although they show a slight warming of the upper troposphere, this warming is less than $1 \mathrm{~K}$. Neither these results, nor those of the PREDICT experiment presented in Smith and Montgomery (2012a), support Raymond and Sessions' hypothesis on the scale of the recirculating pouch region.

In Smith and Montgomery (2012a), we articulated a number of concerns with Raymond and Sessions' hypothesis. In 
a nutshell, we questioned the applicability of a theory for small perturbations to radiative-convective equilibrium formulated on the equator to off-equatorial tropical disturbances possessing relatively small spatial and temporal scales. In the Raymond and Sessions's model, the imposed temperature and moisture perturbations were motivated by observational data taken within a mesoscale convective vortex embryo and proximity soundings of the same mesoscale convective system. It would be fortuitous if these proximity soundings were representative of the radiative-convective-equilibrium state of the entire tropical disturbance, if such a state exists. We are not convinced that a comparison with solely the thermodynamic characteristics of a non-developing disturbance on one day during the TCS08 experiment is physically defensible. Indeed, we believe it needs to be demonstrated whether the thermodynamic control as envisaged by Raymond and Sessions is essential in a rotational environment.

\section{Conclusions}

This work was carried out to determine the salient thermodynamic characteristics of the "pouch region" of developing Typhoon Nuri (2008) that was identified in Part 1. These features are compared with those in similar analyses of developing tropical disturbances observed during the PREDICT experiment during the summer of 2010. Like the PREDICT cases, the genesis of Nuri was spatially and temporally well sampled.

We calculated the mean soundings of virtual potential temperature $\left(\theta_{\mathrm{v}}\right)$, pseudo-equivalent potential temperature $\left(\theta_{\mathrm{e}}\right)$ and relative humidity on four consecutive days from the predepression stage to the Typhoon stage. In the genesis phase (between Nuri1 and Nuri2) the vertical structure of both $\theta_{\mathrm{v}}$ and $\theta_{\mathrm{e}}$ shows little variability between soundings. This variability increases noticeably between Nuri3 and Nuri4. The changes in relative humidity follow broadly the changes in $\theta_{\mathrm{e}}$. The vertical profiles of $\theta_{\mathrm{e}}$ during the genesis show a modest deficit of $\theta_{\mathrm{e}}$ between the surface and the height of minimum $\theta_{\mathrm{e}}$ (between 3 and $4 \mathrm{~km}$ ), of $15 \mathrm{~K}$ to $17 \mathrm{~K}$. These characteristics are all similar to those found for developing disturbances during the PREDICT experiment.

As noted above, neither these results nor those of the PREDICT experiment support the thermodynamic control hypothesis on the scale of the pouch region.

The observations of Nuri described herein provide a context for future study of the moist convective dynamics within the rotating pouch region of a tropical wave critical layer and also the interaction between the pouch region and its environment. Both of these topics are important elements of the marsupial paradigm and research efforts in both directions are currently underway.
Acknowledgements. We are grateful to Tim Dunkerton, Dave Raymond and Ed Zipser for their constructive comments on the original version of manuscript. We would like to thank the US Office of Naval Research for supporting the TCS08 experiment. We would also like to thank Lou Lussier for his expert assistance with the creation of Fig. 2. MTM acknowledges financial support from the US Office of Naval Research Grant No. N00014-03-1-0185 and NSF ATM-0733380. RKS acknowledges financial support for tropical-cyclone research from the German Research Council (Deutsche Forschungsgemeinschaft) from Grant No. SM30/25-1.

Edited by: T. J. Dunkerton

\section{References}

Bister, M. and Emanuel, K. A.: The genesis of Hurricane Guillermo: TEXMEX analyses and a modeling study, Mon. Weather Rev., 125, 2662-2682, 1997.

Bolton, D.: The computation of equivalent potential temperature, Mon. Weather Rev., 108, 1046-1053, 1980.

Chang, C. P., Morris, V. F., and Wallace, J. M.: A statistical study of easterly waves in the western Pacific: July-December 1964, J. Atmos. Sci., 27, 195-201, 1970.

Dunkerton, T. J., Montgomery, M. T., and Wang, Z.: Tropical cyclogenesis in a tropical wave critical layer: easterly waves, Atmos. Chem. Phys., 9, 5587-5646, doi:10.5194/acp-9-5587-2009, 2009.

Elsberry, R. and Harr, P.: Tropical cyclone structure (TCS08) Field experiment scientific basis, observational platforms, and strategy, Asia-Pacific J. Atmos. Sci., 44, 1-23, 2008.

Haynes, P. and McIntyre, M. E.: On the evolution of vorticity and potential vorticity in the presence of diabatic heating and frictional or other forces, J. Atmos. Sci., 44, 828-841, 1987.

Houze, R. A., Lee, W.-C., and Bell, M. M.: Convective contribution to the genesis of Hurricane Ophelia (2005), Mon. Weather Rev., 137, 2778-2800, 2009.

Montgomery, M. T. and Smith, R. K.: Tropical-Cyclone Formation: Theory and Idealized modelling, Report for the Seventh International Workshop on Tropical Cyclones, La Réunion, November 2010, World Meteorological Organization, Geneva, Switzerland, 2011.

Montgomery, M. T., Lussier III, L. L., Moore, R. W., and Wang, Z.: The genesis of Typhoon Nuri as observed during the Tropical Cyclone Structure 2008 (TCS-08) field experiment - Part 1: The role of the easterly wave critical layer, Atmos. Chem. Phys., 10, 9879-9900, doi:10.5194/acp-10-9879-2010, 2010.

Montgomery, M. T., Davis, C., Dunkerton, T., Wang, Z., Velden, C., Torn, R., Majumdar, S., Zhang, F., Smith, R. K., Bosart, L., Bell, M. M., Haase, J. S., Heymsfield, A., Jensen, J., Campos, T., and Boothe, M. A.: The Pre-Depression Investigation of Cloud systems in the Tropics (PREDICT) experiment: Scientific basis, new analysis tools, and some first results, B. Am. Meteorol. Soc., 93, 153-172, 2012.

Raymond, D. J. and López Carrillo, C.: The vorticity budget of developing typhoon Nuri (2008), Atmos. Chem. Phys., 11, 147163, doi:10.5194/acp-11-147-2011, 2011.

Raymond, D. J. and Sessions, S. L.: Evolution of convection during tropical cyclogenesis, Geophys. Res. Lett., 34, L06811, doi:10.1029/2006GL028607, 2007. 
Raymond, D. J., Sessions, S. L., and Lopez, C. L.: Thermodynamics of Tropical Cyclogenesis in the Northwest Pacific, J. Geophys. Res., 116, D18101, doi:10.1029/2011JD015624, 2011.

Reasor, P. D., Montgomery, M. T., and Bosart, L. F.: Mesoscale observations of the genesis of Hurricane Dolly (1996), J. Atmos. Sci., 62, 3151-3171, 2005.

Reed, R. J. and Recker, E. E.: Structure and properties of synopticscale wave disturbances in the equatorial western Pacific, J. Atmos. Sci., 28, 1117-1133, 1971.

Ritchie, E. A. and Holland, G. J.: Large-scale patterns associated with tropical cyclogenesis in the western Pacific, Mon. Weather Rev., 127, 2027-2043, 1999.
Smith, R. K. and Montgomery, M. T.: Observations of the convective environment in developing and non-developing tropical disturbances, Q. J. Roy. Meteorol. Soc., in press, 2012a.

Smith, R. K. and Montgomery, M. T.: How important is the isothermal expansion effect to elevating equivalent potential temperature in the hurricane inner-core?, Q. J. Roy. Meteorol. Soc., in press, $2012 b$.

Tory, K. J. and Montgomery, M. T.: Internal influences on tropical cyclone formation. The Sixth WMO International Workshop on Tropical Cyclones (IWTC-VI), San Jose, Costa Rica, 2006, World Meteorological Organization, Geneva, Switzerland, 2006. 